Situation Normality and the Shape of Search: The Effects of Time Delays and Information Presentation on Search Behavior

\author{
Nolan J. Taylor \\ Kelley School of Business \\ Indiana University \\ Indianapolis, IN 46202 \\ notaylor@iupui.edu \\ 317-274-0185
}

Fax: 317-274-3312

\author{
Alan R. Dennis \\ Department of Operations and Decision Technologies \\ Kelley School of Business \\ Indiana University \\ Bloomington, IN 47405 \\ ardennis@indiana.edu \\ 812-855-2691
}

Fax: 812-855-4985

Jeff W. Cummings

Department of Information Systems and Operations Management

Cameron School of Business

University of North Carolina at Wilmington

Wilmington, NC 28403

jc49@indiana.edu

812-272-1587

This is the author's manuscript of the article published in final edited form as:

Taylor, N. J., Dennis, A. R., \& Cummings, J. W. (2013). Situation normality and the shape of search: The effects of time delays and information presentation on search behavior. Journal of the American Society for Information Science and Technology, 64(5), 909-928. Available from: http://dx.doi.org/10.1002/asi.22782 


\title{
Situation Normality and the Shape of Search: \\ The Effects of Time Delays and Information Presentation on Search Behavior
}

\begin{abstract}
Delays have become one of the most often cited complaints of Web users. Long delays often cause users to abandon their searches, but how do tolerable delays affect information search behavior? Intuitively, we would expect that tolerable delays should induce decreased information search. We conducted two experiments and found that as delay increased, a point occurs at which time within-page information search increases ; that is, search behavior remained the same until a tipping point occurs where delay increases the depth of search. We argue that situation normality explains this phenomenon; users have become accustomed to tolerable delays up to a point (our research suggests between 7 and 11 seconds) after which search behavior changes. That is, some delay is expected, but as delay becomes noticeable but not long enough to cause the abandonment of search, an increase occurs in the "stickiness" of Web pages such that users examine more information on each page before moving to new pages. The net impact of tolerable delays was counter intuitive: tolerable delays had no impact on the total amount of data searched in the first experiment, but induced users to examine more data points in the second experiment.
\end{abstract}

Key Words: Internet, time, delay, service delays, information search, information foraging, stickiness, situation normality 


\section{INTRODUCTION}

From the infancy of the Web, delays have been and continue to be both highly publicized and highly researched (Kehoe, Pitkow, Sutton, Aggarwal, \& Rogers, 1998; Khosrowpour \& Herman, 2000; Lightner, Bose, \& Salvendy, 1996). Delays have been identified as one of the primary sources of user frustration (Ceaparu, Lazar, Bessiere, Robinson, \& Schneiderman, 2004; Forrester, 2007; Rose, Lees, \& Meuter, 2001; Rose, Meuter, \& Curran, 2005) with long delays leading to reduced Web site success (Palmer, 2002) as well as negative attitudes to online retailers (Rose, Evaristo, \& Straub, 2003; Rose \& Straub, 2001). The increased interest in mobile computing technologies (Hong \& Tam, 2006; Lyytinen \& Yoo, 2002; Yoo, 2010) emphasizes the need to have a better understanding of delays as mobile providers continually expand to meet demand and the resulting delays from the increase in demand. While many have suggested that broadband technologies are the best way to reduce delays, this assumes that Web delays are all network related. In many cases, delays can be reduced but not completely eliminated because factors not directly related to the network connection can also impose delays (Galletta, Henry, McCoy, \& Polak, 2004; Rose \& Straub, 2001).

On the network side, traffic continues to match the upgrades in the network bandwidth leading some to predict that delays will continue to be a concern (Sears \& Jacko, 2000). This problem continues to increase as page size and complexity are ever increasing to meet the demands of users (Galletta, Henry, McCoy, \& Polak, 2006). In fact, the increased popularity of broadband connections has caused heavier Internet traffic loads and greater performance requirements as a result of higher user expectations (Leighton, 2009). Compounding this problem is the fact that as delay times have decreased so have the perceptions of what a tolerable delay is. In the 1990s, 8-10 seconds was seen as tolerable (Davis \& Hantula, 2001; Nielsen, 1997; Sliwa, 1999), but a decade later, it has dropped to 2-4 seconds (Forrester, 2007, 2009; Galletta et al., 2004). Thus, whether due to network delays, processing delays, or user perceptions, a site may still consist of several pages with "tolerable" delays. These delays, while noticeable, do not always result in the decision maker abandoning the task but may influence them to change their search behavior.

A variety of theories have been advanced for explaining a user's search behavior. Search behavior involves an individual's interaction with an information system which includes the individual judging the relevancy of the information retrieved (Wilson, 2000). The dominant theories explaining search behavior include motivations for information seeking (Wilson, 1981, 2006), the information search process (Kuhlthau, 2004) and sense-making (Dervin, Foreman-Wernet, \& 
Lauterbach, 2003). These theories examine general search behavior and the various processes employed by the user in seeking information. However, when examining changes to search behavior in the face of delays, one must account for other potential factors due to the greater effort created by that delay. Delays may cause an individual to compensate for the increased search costs associated with the added search time (in accordance with cost-benefit theory). Cost-benefit theory assumes the seeker's motive is to maximize gains (weighted benefits - weighted costs) and the theory suggests that in order to maximize gains, decision makers tend to be effort minimizers. Thus, in the face of increased cost or effort, information seekers tend to adopt effort-minimizing strategies that reduce the extent of information search or reduce the amount of time used to consider the acquired information (Benbasat \& Todd, 1996; Karim, Hershauer, \& Perkins, 1998; Smith, Mitchell, \& Beach, 1978; Teeni, 1990; Vessey, 1994). ${ }^{1}$ Such effort reduction may impair the decision quality because decision makers may base decisions on incomplete information (Benbasat \& Dexter, 1986; Edland \& Svensen, 1993; Hwang, 1994). When applying cost-benefit theory to the Web, it is unclear whether this effort reduction will come in the form of reduced number of pages searched or reduced within-page search. These effort reduction strategies can have markedly different impacts on decision quality.

Information foraging theory (Pirolli \& Card, 1999) may be a useful lens for examining Web search behavior.. Information search behavior involves the "hunt" for information in which information sources in the decision maker's environment vary by value and relevance. The source information can be viewed as "patches" of varying importance. "Informavores" (i.e., information carnivores) seek to maximize the gains of information per unit cost rather than effort reduction (Pirolli \& Card, 1999, p3). From this view, the cost of information search includes both the actual costs of obtaining information as well as the opportunity cost of viewing one piece of information over another. Thus, increases in delays may actually lead to an increase in search due to the increased cost of returning to a page (Dennis \& Taylor, 2006).

Our current study examines the effects of different amounts of delay during Web search to understand the relationship between the delay amount and the nature of information presentation on user search behavior. The idea of changes in search behavior due to delays has been previously posited by Dennis and Taylor's (2006) examination of "acceptable" Internet delays. They found that, when faced with tolerable delays, users examined more information while decision quality and decision time remained unaffected. While these results are useful, additional questions remain unanswered. Do delays have linear (Palmer 2002) or curvilinear effects (DiClemente and Hantula 2004) on search behavior? Is there a 
point at which search behavior will change due to delays? In this paper, we use the lens of situational normality to argue that the impact of delay on search behavior is neither linear nor curvilinear, but rather follows a step function: delay has no impact on search behavior until some threshold is reached and is no longer perceived as "normal," at which point behavior changes. Thus, in the context of information foraging theory (Pirolli \& Card, 1999), the forager searching for information on a website will not change their search behavior until there is a change in their environment (i.e. the search environment is no longer perceived to be "normal"). This change is caused by increased Internet delay resulting in a change to search behavior.

Additionally, we examine the impact of information presentation in conjunction with Web delays on search behavior in the presence of delays. Web information can be presented by alternative (e.g., each Web page presents all the information about one product) or by attribute (e.g., each Web page presents information for all products on one dimension (e.g., price, weight)). Dennis and Taylor (2006) focus on a "by attribute" presentation which tends to be atypical of most current Web sites. Many sites today present information by alternative in which all the information for one specific product is presented with links to alternatives. Prior research has examined the difference between information presentation from a technology acceptance perspective (e.g. ease of use, usefulness, etc.) (Kamis, Koufaris, \& Stern, 2008). However, limited research has examined information presentation from a search perspective, especially in the face of delays. Understanding how information is presented is important as we have moved to highly connected society and maintaining this degree of connectivity may be directly tied to navigability and information architecture on websites (Morville, 2005). Using two different conditions, the experiments reported here show the effects of delay on search behavior when information is presented either by attribute or by alternative. This provides further insight into how presenting information in different formats (i.e. alternative or attribute) has the potential to impact decision quality beyond the lens of delay.

In the subsequent sections, we start our discussion by examining delay and information search. Many factors may affect a user's choice when navigating the Web (e.g. technological, personal, contextual, etc.), but we focus on the effects of just one factor: response time delay as that is cited as one of the most frustrating features of the Web (Ceaparu et al., 2004). We posit that situation normality provides a theoretical lens into understanding how users react to these delays. Under situation normality, we suggest a user's search behavior will remain unchanged until a "tipping point" occurs (i.e. a point at which search behavior changes in response to an increased amount of delay). This is followed by how delays can 
affect search behavior by changing the perceptions of the cost and effort required to perform the task. An additional experiment is introduced to further understand the tipping point that often occurs in the face of delays as well as the impact information presentation may have search behaviors. Finally, a general discussion and implications are discussed.

\section{ONLINE INFORMATION SEARCH:}

\section{Service Delays and Situation Normality}

Understanding how delay impacts search behavior is important as web developers are designing sites to become more complex and more interactive to meet the increased demands of users (Leighton, 2009). This results in developers balancing complexity/interactiveness of the site with loading times which may cause increased delay, especially as the world moves to less powerful mobile devices operating over lower bandwidth circuits.

Actual delay times can impact user behaviors, but it is the user's perception of time that has the greatest impact on behavior (Katz, Larson, \& Larson, 1991). Different users may have different perceptions of the expected amount of time a search should take and these perceptions can affect their information seeking behavior and how they evaluate information and make choices (Bellman, Lohse, \& Johnson, 1999; Ozmutlu, Spink, \& Ozmutlu, 2003; Slone, 2007). Because individuals may alter choices based upon time for conducting a search, understanding the specific points at which time delay impacts search behavior has the potential to have financial implications. Thus, for any specific objective measure of time delay, the subjective interpretation of that delay (whether long or short) will vary from individual to individual and context to context (Katz et al., 1991).

The relationship between delays and their subjective interpretation has been approached from both a linear and a curvilinear perspective. A linear relationship is often assumed where researchers focus on situations in which only delay and no delay is examined (Dennis \& Taylor, 2006; Galletta et al., 2006). Other research has suggested a linear relationship exists with delay. As download delays are reduced, perceived success increases (Palmer, 2002). Palmer argues that increased delays will have a linear effect on user perceptions of Web sites as well. Thus increasing delays (e.g. 3 seconds, 5 seconds, 7 seconds, etc.) will continue to have primarily a linear effect on attitudes and satisfaction.

An alternative suggestion is a curvilinear relationship; miniscule delays (e.g., less than 2 seconds) are often overlooked but as delay increases, perceptions of delay change in a curvilinear manner (Galletta et al., 2004). DiClemente and Hantula (2003) found delay caused a curvilinear effect in which increased delays caused a reduction in both percent 
of purchases and time spent in a store up to 8 seconds of delay at which point increasing delay no longer affected purchases or time in a store. These curvilinear effects from delay have also been found in subsequent studies examining delay's influence on attitudes, purchasing decision and satisfaction (Galletta et al., 2004; Hantula, Brockman, \& Smith, 2008; C. Smith \& Hantula, 2003).

We argue that delay's effect on search behavior is neither linear nor curvilinear. Instead, we posit a step function relationship; that is, as delays increase, no change occurs until a tipping point is reached at which point search behavior changes. Step functions have been observed in a variety of social phenomena. For example, a tipping point called a separation threshold has been identified in technology professionals' job retention (Josefek Jr. \& Kauffman, 2003). Below the separation threshold, an employee intends to remain with his/her current employer. However, once a variety of job factors, such as dissatisfaction and unmet work-related expectations, surpasses this separation threshold, the employee decides to leave his/her job (Josefek Jr. \& Kauffman, 2003). In this example, there is no linear or curvilinear relationship between the independent and dependent variables; instead, they follow a step function. This is also similar to the concept of a process model in behavioral research in which behavior follows one pattern until a tipping point is reached whereupon the behavior experiences a state change and then follows a different pattern (Shaw, 1997).

We believe that situational normality is the trigger for the delay step function in Web search. Normality is based on a perception of "how things ought to be" and is the centrepiece of understanding what is normal and what is not normal (Misztal, 2001). Normality is the perception that things are in the proper, usual and customary order (Lewis \& Weigert, 1985). Thus, situational normality is the belief that things appear to be normal within a specific situation (Garfinkel, 1963). Beliefs about what constitutes situational normality develop overtime as individuals interact within a specific situation and form beliefs about what is proper, usual and customary about the interactions occurring in that situation. There is a perception of regularity and reliability (Misztal, 2001) and if interactions do not deviate from these patterns, a sense of what is normal within a given situation develops. In general, individuals will continue to hold normality beliefs concerning a situation (unless events occur that lead them to believe otherwise), leading them to assume a normal interaction will occur (Baier, 1986; Garfinkel, 1963).

Within an online environment, research has focused on how situations appear to be normal from a website interaction perspective. Individuals exhibiting high levels of situation normality perceive the Web environment to be an orderly, 
appropriate place to conduct business (McKnight, Choudhury, \& Kacmar, 2002). These users have a higher level of trust based upon the belief that everything is in proper order compared to their past interactions in similar situations.

We posit users form general beliefs concerning delays for Web search (i.e. situational normality). That is, most users have a belief about what is a "normal" amount of delay for Web searches, based on their past experiences with the Web. Researchers continue to suggest that every Web user faces some delay on a regular basis (Galletta et al. 2004b; Rose et al. 2001), prompting us to suggest that individual users have come to accept that some delay will normally exist when they interact in an online environment. For example, users conducting a search on the Web may not notice minimal delays as they have had previous interactions with the Web environment and over time, have found a small delay to be normal. However, when delay reaches a point at which it is no longer perceived to be "normal", users may change their search behaviors. Thus, situation normality would suggest that tolerable delays will remain unnoticed and have no effect on behavior. But once the delay becomes a large enough trigger a change in the perception of situational normality, it will trigger a change in search behavior (i.e. a step function relationship).

Such a step function relationship based on situational normality would suggest that search behavior will not change until a tipping point is reached. This is different from prior linear or curvilinear relationships suggesting search behavior will change in which delay is hypothesized to continually change search behavior as delay increases. If delay does follow a step function relationship as proposed through situation normality, it would suggest that some delay would have little impact on behavior, thus allowing developers additional freedom to increase the interactiveness demanded by users while not impacting the user's search behavior (Leighton, 2009).

\section{Information Search on the Web: Satisfaction and Effort}

Although users may assume a certain amount of delay to be normal, it is not fully understood how and to what extent might these delays impact individual satisfaction and effort. Web delays have been noted as a key source of dissatisfaction. Download times of 30 seconds have been associated with negative evaluations (Dellaert \& Kahn, 1999) with delays as little as 13 seconds rated as "long" causing reduced satisfaction and search abandonment (Fui Hoon Nah \& Kim, 2000). More recently, a number of other studies have investigated responses to tolerable waiting time (TWT) on the Web and collectively have confirmed this delay-dissatisfaction link (Dennis \& Taylor, 2006; Galletta et al., 2004; Fiona H. Nah, 2004; Palmer, 2002; Rose et al., 2003; Rose \& Straub, 2001; Weinberg, 2000). McKinney et al (2002) suggest that 
one factor of user satisfaction within online environment is directly linked to the specific site's performance (i.e. how well information is delivered). Thus, when a site is unable to effectively provide information in a timely manner, satisfaction will be impacted leading to negative consequences such as abandonment of search or switching to another, potentially competitor's site (Forrester, 2009). This can be detrimental in an online store environment in which satisfaction with experience is often tied to buyer's returning to the website in the future which impacts future sales. Therefore, balancing delay and user tolerance is important to overall satisfaction. Under the effects of situation normality, we argue that tolerable delays will remain unnoticed, not influencing user's perceptions until delay reaches a tipping point at which satisfaction is affected. Therefore, we hypothesize:

\section{H1: As response time delay increases, satisfaction will remain unchanged until a tipping point is reached, after which satisfaction will decrease.}

Delay increases the real and - more importantly - the perceived cost of information search because search takes longer as delays increase. Even with a dampening effect from a number of factors previously shown to provide positive search outcomes (e.g. Web site familiarity and reducing page search levels (Galletta et al., 2006)), there is still a perceived cost associated with delays. As the cost of information search due to delay increases, decision makers often trade off the amount of effort to be spent with the benefits expected (Beach \& Mitchell, 1978; Peter, Todd \& Benbasat, 1999). They can react in one of two basic ways to increasing search costs (Todd \& Benbasat, 1992; Peter, Todd \& Benbasat, 1999).

At one extreme, a decision maker could place primary importance on decision accuracy rather than decision effort. In the face of increasing information search costs, the decision maker valuing accuracy would not change his or her information search behavior but would simply extend total decision time. That is, information search (i.e., the shape and amount of data examined) would not differ significantly between modest delayed and non-delayed cases - and thus neither should decision accuracy - but the amount of time taken would increase. For example, in this case, an online information searcher will continue the task of searching for information when faced with modest delay because this individual wants to make the most accurate decision possible based upon the information available (e.g. this individual may be considering an expensive purchase such as a car). However, this does not come without a cost; it produced a longer information search, resulting in additional time over someone not as concerned with accuracy.

At the other end of the spectrum, a decision maker could place primary importance on the amount of effort expended, rather than decision accuracy. In the face of increasing search costs, the decision maker valuing effort would change 
information search behavior to reduce effort or decision time. That is, information search (i.e. data examined) would be significantly reduced when there is a tolerable delay and thus decision accuracy could suffer. An example of this individual would be the opposite from our previous example. This type of person is not concerned with overall accuracy in their decision, choosing instead to minimize effort or at least constrain effort within some bounds (e.g., I have only 15 minutes to find the information I need). Thus, when faced with delays, this person would not search the same amount of information (compared to one emphasizing accuracy) but instead, would choose a searching behavior that requires less effort.

Previous research suggests that most decision makers typically place more value on effort expended than potential benefit received, except for very important decisions (Einhorn, Hogarth, Demski, \& Swieringa, 1981; Kleinmuntz \& Schkade, 1993; Schkade \& Kleinmuntz, 1994). Even though there is still a need to retrieve information in the decision making process based on the decision maker's deeply seeded need for information (Cole, 2011), decision makers will tend to reduce effort rather than maximize accuracy (Todd \& Benbasat, 1992; Peter, Todd \& Benbasat, 1999). In the face of increased costs of information search due to delays, they tend to adopt effort-minimizing strategies that reduce the amount of information examined before making a decision (Benbasat \& Dexter, 1986; Benbasat \& Todd, 1996; Edland \& Svensen, 1993; Hwang, 1994; Smith et al., 1978; Todd \& Benbasat, 1992). Therefore, decision makers strive to spend a consistent amount of time and effort regardless of whether they are faced with tolerable delays or not ${ }^{2}$. Therefore:

H2: Decision time will not be affected by the magnitude of tolerable response time delays. H3: Perceived effort will not be affected by the magnitude of tolerable response time delays.

\section{Information Search and Information Foraging on the Web: Shape and Outcomes}

Prior research on information search has examined situations in which information is presented in one large pool (e.g., Cook, 1993; Todd \& Benbasat, 1992; Peter, Todd \& Benbasat, 1999). Retrieving one data point costs about the same as retrieving another (i.e. the cost to access any specific piece of information is uniform). However, on the Web, information is typically organized in pages or subsets of information. Rather than retrieving information directly, decision makers typically access information one page at a time. Once a page is displayed, decision makers have access to all the information it contains. Therefore, acquiring data located on another page costs more than acquiring data on the currently displayed page, so information search costs are not uniform. 
Information foraging theory has been advanced as an explanation of information search choices when the cost to retrieve information is not uniform (c.f. Pirolli \& Card, 1999; Spink \& Cole, 2006). Rather than assuming the uniform cost of effort suggested by economic theory (Pollay, 1970), information foraging theory assumes greater complexity in both the information environment and cost perceptions. Foraging for information on the Web and foraging for food in the wild share several features. Resources tend to be unevenly distributed in the environment (currency), all foragers have limited time and experience opportunity costs by choosing to exploit one resource over another (constraints), and uncertainty and risk characterize resource procurement (Sandstrom, 1994, p420). The decision maker then decides on a sequence of pages (strategy) based on varying information value, time constraints, and uncertainty.

Though there are a numerous decisions the forager must make to achieve this optimal prey mix, the two principle decisions include what to consume (prey choice and diet breadth), and where to hunt (time allocation and patch choice). Inclusion in a forager's diet is informed by the information's "scent" - perceptions of the value and cost of information (Pirolli \& Card, 1999). Information scent has been shown to be a valuable determinant of a forager's performance affecting their Web site specific attitudes (Moody \& Galletta, 2008). With a strong scent, a forager can make the correct decision about whether information should be "consumed" (Sundar, Knobloch-Westerwick, \& Hastall, 2007). Otherwise, the consumer may choose to ignore it or consume it on the basis of opportunity costs ("it might be important so l'd better read it"). The information diet model suggests that foragers will favor prey with the highest profitability (benefit - cost). Likewise, where to hunt involves a similar set of decisions. Once in a "patch" a forager must decide whether to continue to hunt within a patch or seek prey in a different patch. Strategies range from sampling all patches to determine the most profitable ones (i.e., "matching") to foraging a single patch until it appears more profitable to hunt elsewhere (i.e., "momentary maximization") (DiClemente \& Hantula, 2003).

The cost of information search is not uniform in the presence of delays. In a situation where delay is miniscule, the forager may follow a "berrypicking" strategy or take the approach of sampling and selecting information to find what is important during the searching process (e.g. moving from page to page freely) (Bates, 2002). This would be considered "diet breadth" under foraging theory. However, cost includes the cognitive effort of such tasks as keeping track of the information examined, the location of the information not yet examine, etc. as well as the psychological stress from the perceived loss of control (Trimmel, Meixner-Pendleton, \& Haring, 2003). Because delays increase the time to move from 
page to page, the cost to search a number of pages will continue to increase with every additional page viewed. Thus, each page is considered to be a "patch" of information with a decision maker viewing each patch as more or less important given a specific situation. Therefore, under this model, a delay would essentially represent an "entry fee" for each page retrieved. This information foraging "patch model" requires an additional "entry fee" be paid by the information forager when moving from page to page as well as additional fees if he or she backtracks to previously viewed pages (Dennis \& Taylor, 2006). Thus, the entry fee to move from page to page caused by delays may trigger a perceived change in the situation (i.e. search process).

As decision makers will often return to previous pages, backtracking in the presence of delays may cause the fee to view a particular page to double, triple or even quadruple depending upon the number of times a searcher returns to the same page ${ }^{3}$. The decision maker's search changes overtime as the both the cost and relevancy of the information being searched changes with delay (Bates, 1989). In this case, the overall situation (i.e. a forager searching information under tolerable delays) changes which is reflected in the search behavior of the decision maker. Thus, delays increase the cost of performing a task by extending the time required to complete it.

For example, many sites are organized by alternative, requiring shoppers to view a new page for each product, which means that delays caused by moving from page to page will increase the time it takes to compare products. With minimal delays, foragers are likely to overlook this cost as minimal delays are seen as normal. Only at the point at which delays are longer than expected and forager ceases to perceive that normality exists, will foragers recognize these costs and change their behavior to minimize these costs. Because decision makers tend to adopt effort minimizing strategies (Benbasat \& Todd, 1996; Smith et al., 1978; Todd \& Benbasat, 1992), the increased cost (i.e., time) of accessing Web pages beyond tolerable response time delays should induce decision makers to reduce the number of Web pages they search. Thus,

\section{H4: As response time delay increases, the number of pages searched will remain unchanged until a tipping point is reached after which the number of pages searched will decrease.}

Information foraging theory suggests that the forager will continue to search within a page of information as long as the marginal benefit exceeds that the marginal cost of moving to a new patch of information (i.e. another Web page) (Pirolli \& Card, 1999). For example, a forager gathering information concerning the purchase of a new technology device will gain a greater benefit by examining as much information on the current page as possible when faced with increased delays of 
moving to another site discussing the item. This is due to the "cost" of time incurred by moving to an alternative page compared to the amount of information gained from the current page. Thus, when faced with delays, the information forager should continue to gather additional information on the current page before incurring the additional cost associated with moving to a new page. The argument that delays actually increase information search may seem counter-intuitive, but one must understand typical search behavior associated with "backtracking" (i.e., to return to a prior page). Backtracking is a common search pattern to gather information previously on a prior page (Charland \& Leroux, 2011). Delays will increase the cost of backtracking resulting in fewer alternative comparisons (i.e., revisiting known information) (Saad, 1998).

The decision of whether to examine a previously viewed page (i.e. backtrack) is a function of both the cost to backtrack and value of information on that particular page. As delay increase beyond what is assumed to be normal (i.e. situational normality no longer present), information foragers should spend extra time and effort in acquiring information during the first visit (as "insurance" against backtracking) to minimize the need to backtrack (Pirolli and Card (1999) offer a mathematical presentation of this argument). Therefore:

H5: As response time delay increases, the amount of information examined per page will remain unchanged until a tipping point is reached after which the amount of information will increase.

The adoption of effort reducing strategies can have a number of undesirable consequences. Delays have been shown to induce decision makers to switch to simpler, non-compensatory decision rules (Payne, Bettman, \& Johnson, 1993) and to significantly reduce decision making performance (Ahituv, Igbaria, \& Sella, 1998; Durrande-Moreau, 1999; Fui Hoon Nah, 2000). In the present case, we posit that decision time will not vary with delay (H3). As the time is "eaten away" by delays considered to be outside a decision maker's sense of normalcy, the net result is decision makers should make poorer decisions by examining fewer alternatives (i.e. a potentially good alternative may be overlooked) as fewer pages are searched. Therefore:

H6: As response time delay increases, decision quality will remain unchanged until a tipping point is reached after which quality will decrease. 


\section{EXPERIMENT 1: Information Presentation by Alternative}

\section{Participants}

123 students from an introductory information systems class at a large state university participated in this experiment. Males comprised $68 \%$ (73) of this sample. As a participation incentive, each participant received $1 \%$ extra credit. As a performance incentive, participants were instructed that the person arriving at the correct choice in the shortest amount of time in each treatment would receive a $\$ 50$ prize.

\section{Treatments}

Participants were randomly assigned to one of four between-participant factorial design treatments that imposed different delay times $\left(0,4,7\right.$, or 11 seconds $\left.{ }^{4}\right)$. The question of what constitutes a "long" delay and a "tolerable" delay has been posed in prior research. This research suggests that the threshold between long and tolerable delays for Web page transitions is in the range of 7-10 seconds. From a cognitive standpoint, 7 seconds represents the upper bound of working memory (Card, Moran, \& Newell, 1983) with previous research suggesting this mark to be seen as a "tolerable" delay (Dennis \& Taylor, 2006). Practitioners have suggested 10 seconds (Nielsen, 1997), the oft-quoted "8 second rule" (Davis \& Hantula, 2001; Sliwa, 1999) and times in between (e.g. 8.57 seconds (Bhatti, Bouch, \& Kuchinsky, 2000)) as thresholds for long delays. Similarly, Shneiderman (1983) and Davis and Olson (1985) argue that a reasonable upper bound for response time is 10 seconds. Delays of less than $8-10$ seconds in accessing individual pages have been interpreted as perceptible but tolerable delays (Newell, 1990; Nielsen, 1993) with quality ratings of Web sites minimally effected below the 8 second mark (Bouch, Kuchinsky, \& Bhatti, 2000). Additionally, one report suggests user expectations for page rendering at 4 seconds (Forrester, 2007), with some cases suggesting 2 second delays impact behavior (Forrester, 2009; Galletta et al., 2004). The goal in selecting specific time delays used in this experiment was to have a range of potentially "tolerable" delays. Because 11 seconds exceeds the suggested upper bound of a tolerable, this would be considered a long delay in the current experiment.

\section{Task}

Participants were asked to select the best digital camera from a set of 20 cameras for use by a department that had a defined set of selection criteria. In this way, the task resembled both a consumer task involving relatively infrequent, high- 
value decisions (e.g. major purchases or vacation planning) as well as a business task where a number of Web sites/online databases need to be consulted in order to select the best alternative from a group of similar choices.

A Web site was designed to present camera information and to record page search data. Several camera ratings sources including Consumer Reports, shopping.com, and epinions.com were consulted in the design of the camera attributes used in this task. We designed the task such that camera attributes specified as high importance in the task were also those that these sites identified as the important criteria used in the selection of a camera for personal use.

Participants were provided with information on 16 attributes for each of the 20 fictitious cameras. The task explicitly assigned each of the attributes one of three weighting values reflecting different importance to the decision. Of the 16 attributes, 4 were assigned a high importance weighting value of 70 , making them very important to the decision; 8 had a medium weighting value of 25, and the remaining 4 had a low importance value of 5 (see Appendix 1 for the list of attributes and weights). Of the 20 alternatives, one alternative was constructed to be the best choice based on its superior performance on a combination of high and medium value attributes. Appendix 2 provides selected portions of the task materials. From these attributes, 20 alternatives were created based on actual attributes of several popular name brand digital cameras.

As is typical on the Web, the experimental Web site presented the camera information organized by alternative in 20 pages with one page for each camera, labeled camera A, camera B, camera C, etc. The information was organized with a "home page" that linked to the information pages for the 20 cameras. Clicking on the link for a camera presented the user with a page displaying the values of the attributes for that camera. After returning to the home page, the user could click links to view other camera pages. For each page examined, participants were free to use any and all information displayed on that page. Participants were given a paper worksheet to write down the information that they wanted to use. The worksheet consisted of a single sheet of paper with all of the alternatives listed down the left margin with space on each row for recording attribute information.

Imposed delays $(0,4,7$, or 11 seconds) were implemented via dynamic HMTL (DHTML). In each case, the page title initially appeared. The remainder of the information on each downloaded information page was initially invisible then made visible at the expiration of scripted timer. Imposed delays were implemented via a script that executed in each information 
page prior to its display. Thus, the delay only occurred between transitions from the home page to an information page (i.e., one direction). In the opposite direction (to home page), the page appeared immediately.

\section{Dependent Measures}

Dependent variables included measures in six separate areas: decision time, decision quality satisfaction, perceived effort, number of pages searched, and the amount of information examined per page.

The decision time was the time participants spent making their decision (after removing the 4, 7, or 11 second system imposed delay for each page visited). Decision quality was measured via a single item coded as 1 for a correct solution and 0 for an incorrect solution.

Satisfaction was assessed via a post-session questionnaire using four 7-point items taken from McKinney, Yoon, and Zahedi (2002). Cronbach's alpha was .92. Perceived effort was measured via a post-session questionnaire using a modified version of the NASA-TLX (NASA Task Load Index) developed by Fisher and Ackerman (1998). Cronbach's alpha for the six-item construct (7-point scales) was .88. Appendix 3 provides the items.

The number of pages searched was measured using the number of pages examined as recorded in the computer logs. This figure was calculated by counting all of the information pages accessed, (not counting multiple accesses of the same page); that is, how many of the 20 unique pages did the participant examine (recall that there were 20 pages in total, one for each camera). The amount of information examined per page (the number of data points examined per page) was calculated by counting the number of data points written by each participant on a paper worksheet they received to assist them in performing the task. The total number of points recorded was divided by the number of pages to produce the average number of points examined per page. The validity and reliability of using this paper worksheet to record data point level search are discussed in Appendix 4.

\section{Procedures}

Participants performed the task in groups of up to 16 persons with each group part of the same delay treatment. Within each experimental group, computers were separated by cubicles so that no participant was able to see the progress or the search strategy of other participants. Participants signed up for a 90 minute sessions of their choice but were free to go at any time after they completed the post-session questionnaire. 
Training and practice were included prior to the task as suggested by previous decision support systems research (Jarvenpaa \& Dickson, 1989). Participants were first trained to use the Web site by completing an apartment selection problem requiring them to use the browser similar to that required for the experimental task. The Web site used in the practice task exhibited the same delay time as the experimental treatment to which a participant was assigned. The duration of the practice session varied between participants, as participants were instructed to take as much time as they wished to become comfortable with the system (typically 7-12 minutes).

Once participants completed the practice session, they proceeded with the experimental task. Participants were not informed of any time expectations, but all finished in less than 45 minutes. After recording their decision, participants completed a questionnaire, were debriefed and released.

\section{Results}

As a manipulation check, we asked participants (on the post-session questionnaire) to estimate the perceived delay. Mean perceived delays for the $0,4,7$, and 11 second treatments were 1.4 seconds, 3.3 seconds, 7.1 seconds and 7.5 seconds, respectively. An ANOVA analysis of perceived delays found a significant main effect for treatment $(F(3,119)=$ $34.1, p<.001)$. Planned comparisons indicated statistically significant differences between all delay treatment pairs $(p<$ .05) except for 7 and 11 seconds ( $p=.638$ ). The results suggest a successful delay manipulation.

Multiple analysis methods were employed. A multivariate analysis of variance was conducted using all dependent variables (except decision quality, a binary dependent variable). Follow-up ANOVAs were used after the MANOVA to identify individual effects. Decision quality was analyzed using cross tabulations (crosstabs). Table 1 presents means and standard deviations. A MANOVA on the entire group of dependent variables (except for decision quality) found significant effects due to delay (Wilks' $\lambda=.787, F=1.92, p=.021$ ), so follow-up ANOVAs were conducted.

Hypothesis $\mathrm{H} 1$ posits that following a tipping point, satisfaction decreases as delay increases. An ANOVA found a significant main effect $(F(3,119)=3.23, p=.025)$ for delay. A post-hoc analysis of the mean differences across the treatments (i.e. times) was conducted using a Ryan-Einot-Gabriel-Welsch F test to understand how the specific treatments were different from one another; this is a standard post-hoc test available in the SPSS GLM procedure that is statistically more powerful than other post-hoc tests (SPSS,2007). This analysis found those in the zero-delay treatment to be more 
satisfied than those in the 11-second delay treatment, but no other difference, suggesting the tipping point occurs somewhere between 7 and 11 seconds. Thus, $\mathrm{H} 1$ is supported.

\section{Insert Table 1 here}

Hypotheses $\mathrm{H} 2$ and $\mathrm{H} 3$ argue that that delay will have no effect on decision time and perceived effort. An ANOVA analysis found no significant effects on decision time due to delay $(F(3,119)=1.66, p=0.179)$. Although the amount of perceived effort appeared to increase with increasing delay, the relationship was not statistically significant $(F(3,119)=2.49$, $p=0.064)$. As an aside, we note that the power to detect a medium effect size with 30 participants per treatment was .98 . That is, if a relationship exists between delay and the dependent variable, there is a $98 \%$ chance that it will be statistically detected. Together hypotheses $\mathrm{H} 2$ and $\mathrm{H} 3$ results are consistent with an effort minimization perspective as found in prior research.

Hypothesis $\mathrm{H} 4$, that the number of pages examined would decrease with increased delay, was supported $(F(3,119)=2.90, p=0.038)$. A Ryan-Einot-Gabriel-Welsch $F$ test found those in the 11-second delay treatment to examine fewer pages than those in the 7-second and those in the zero-delay treatments, but no other differences (H4 supported). Again, these results suggest that a tipping point occurs somewhere between 7 and 11 seconds.

ANOVA analysis on the number of data points per page examined found a significant difference due to delay $(F(3,119)=2.85, p=.040)$. A Ryan-Einot-Gabriel-Welsch $F$ test found those in the 11-second delay treatment to examine more points per page than those in the other treatments, but no other differences. $\mathrm{H} 5$ is supported in which behavior changed at the 11 second delay treatment (see Figure 1). Analysis of decision quality (H6) revealed no statistically significant differences in the mean number of correct solutions per treatment $\left(X^{2}(3)=5.16, p=.161\right)$. Thus hypothesis $H 6$ is rejected.

\section{Insert Figure 1 here}

\section{DISCUSSION}

The pattern of results in Experiment 1 is generally supportive of the situation normality view of information search behavior, suggesting that it is a useful explanation of Web search behavior in the face of delays. Participants did not spend significantly more time or effort as delays increased until a tipping point was reached. At this point, delay was no longer considered to be normal causing search behavior to change. As delays increased, participants examined fewer pages but 
more data points per page. Thus, while prior research in non-Web environments would suggest a reduction in search efforts in the face of delays, our results suggest a redistribution of search efforts: increased within-page search and decreased between-page search. While such increased within-page search may be counter intuitive, it is consistent with information foraging theory. Because the cost to move among pages is higher with greater delays, information foragers search longer within pages.

Our results show a step function in the perception of delays and their impact on behavior, suggesting that users expect some delay as normal when searching the Web to a "tipping" point (i.e. situation normality). Delays of 4 and 7 seconds reduced satisfaction somewhat similarly compared to the no delay treatment. Delays of 11 seconds further reduced satisfaction and also changed search behavior. This suggests that information foragers experience a tipping point after which delays caused a change of search behavior from the 7 second delay to the 11 second delay. Between these delays, a difference in both search behavior and satisfaction was observed. Interestingly, the delay treatments that were perceived to be insignificantly different (i.e. 7 and 11 seconds) appear to be the point at which we see significantly different behaviors from information foragers. This supports our argument that users experience situation normality surrounding tolerable delays on the Web. In other words, when delays are perceived to be "normal," search behavior remains unaffected. However, when delay reaches a point at which it is perceived abnormal (i.e., between 7 and 11 seconds), users react by changing their search behavior.

Information search costs may also differ based upon how the information is presented to searchers (e.g. products presented by attribute or alternative). When searching for product information on the Web, sites such as Amazon.com may offer alternatives to the current product with each located on a different page requiring one to move from the originally searched product. Other sites are organized by attributes where there is a page listing the best products based on specific attributes (e.g. consumer product comparison sites). Thus, information presentation on Web sites may also differ, and potentially affect search behavior when delays are present.

Information on the Web is usually spread across multiple pages. Typically, a single main Web page contains links to a variety of information located across other pages or potentially, different Web servers. From a single page, users have the opportunity to click on a link to another page providing, for example, more information about alternative(s) as they shop (e.g. Amazon.com). The information on these pages can be organized in different ways. All information about one product, 
for example, can be on one Web page (a by alternative presentation), so the user can navigate from page to page gathering information about each product in turn. Alternately, the information can be organized so that each page contains information on one attribute for all products, such as price or weight (a by attribution presentation).

\section{Search Behavior on the Web and Situational Normality with Delays and Non-uniform Page Values}

In Experiment 1, each page contained information on a single alternative. In this case, each page essentially had the same potential value because, a priori, each camera had an equal likelihood of being the correct choice (on first page visit). However, in many cases on the Web, different pages have different values to the decision, depending on how the information is presented (Yuan \& Belkin, 2010b). For example, Web search engines typically list results in order of relevance. Thus, links at the top of the page are more "valuable" than links at the bottom. Other methods include sales rank ordering (e.g., www.barnesandnoble.com/gateway/bestsellers.asp), number of visits (e.g., www.cnn.com/mostpopular), and customer ratings (e.g., www.imdb.com/chart/top). It is unclear how differing page values affect the perceptions of situation normality and search behavior. Experiment 2 attempts to answer these questions by specifically attaching values to the data pages. We again use information foraging theory to predict the search behavior impacts in the presence of delays.

Much like a foraging animal in the wild must select a mix of prey with varying value; the decision maker must decide which information to pursue depending upon its value. Information foraging "diet selection" models are concerned with determining the information diet such that the rate of gain of relevant information is optimized. Information profitability (the value of information returned per unit of time) drives which information is pursued as well as which patches are foraged (Pirolli \& Card, 1999). If one chooses to focus on less profitable items, the opportunity to go after more profitable items will be missed.

As in nature, certain "dietary elements" may be indispensable (high profitability) and thus simply substituting a greater number of lower profitability items may not be an option, no matter how abundant substitutes are in the environment (Pirolli \& Card, 1999). This same pattern should be present in Web searches. Because delays don't change the distribution of indispensable information, selection of which high value patches (pages) to pursue should be more or less unaffected by between-patch delays. Alternatively, search of lower importance will remain unchanged to a tipping point at which 
searching of these pages is reduced. Thus, under increased delay costs, total number of pages searched is reduced while pages deemed as essential for the decision maker to examine should remain unchanged.

H7a: Decision makers will examine the same number of high importance pages regardless of tolerable response time delays.

$\mathrm{H} 7 \mathrm{~b}$ : As response time delay increases, search behavior will remain unchanged until a tipping point is reached after which decision makers will examine fewer lower importance pages.

EXPERIMENT 2: Information Presentation by Attribute

\section{Design}

The design of Experiment 2 closely mirrored the design of Experiment 1, with the exception of the manner in which the information was organized and the delay times used. From the previous experiment, there appears to be a tipping point in which search behavior did not significantly change until somewhere between the 7 to 11 second mark. Because no important differences were found between the 4 and 7 second treatments in Experiment 1, the 4 second delay treatment was omitted in this experiment.

138 students at a large state university participated. Males comprised $70 \%(90)$ of this sample. As a participation incentive, each participant received the same extra credit points. As a performance incentive, the participant arriving at the correct choice in the shortest amount of time in each treatment received a $\$ 50$ prize. Participants were randomly assigned to one of three delay treatments $(0,7$, or 11 seconds) in a between-participant factorial design.

Participants were instructed to select the best digital camera from the same set of 20 cameras. A Web site similar to that used in Experiment 1 was designed, but differed in the manner in which the information was organized. In Experiment 2, the information pages organized the camera data by the 16 attributes. Each information page contained information on all 20 cameras for that particular attribute. For example, the optical zoom page listed the optical zoom levels for all twenty cameras. The home page listed the 16 attributes grouped by importance level.

The dependent variables in Experiment 2 were identical to those of Experiment 1 (i.e., satisfaction, decision time, perceived effort, number of pages accessed, points considered and decision quality) with the addition of the number of pages in each of the three attribute importance categories (see Appendix 1). That is, how many of the four high importance pages, eight medium importance pages, and four low importance pages were accessed. Reliabilities for the questionnaire 
measures (satisfaction and perceived effort) were again adequate (.89 and .90 , respectively). Analyses were conducted in a similar manner to Experiment 1. For hypotheses $\mathrm{H} 7 \mathrm{a}$ and $\mathrm{H} 7 \mathrm{~b}$, comparison of the number of pages accessed by value was performed using repeated measures ANOVA.

\section{Results}

Participants again were asked to estimate the perceived delay time. Mean delay estimates for the 0,7 , and $11 \mathrm{sec}$ treatments were 1.6 seconds, 5.7 seconds, and 7.0 seconds, respectively. Analysis of the measure of estimated delay revealed a significant main effect for imposed delay $(F(2,137)=29.2, p<.001)$. Planned comparisons indicated statistically significant differences between all delay treatment pairs $(p<.05)$. The results suggest a successful delay manipulation. Table 2 presents the means and standard deviations. An overall MANOVA found significant effects due to delay (Wilks' $\lambda$ $=.850, F=2.21, p=.017)$, so follow-up ANOVAs were conducted. Satisfaction was again found to be related to delay $(F(2,135)=4.78, p=.010)$; a Ryan-Einot-Gabriel-Welsch $F$ test found those in the zero-delay treatment to be more satisfied than those in other treatments, but no differences between 7-second and 11-second delay treatments. Thus, this suggests the perception of situation normality changed from the previous experiment, occurring sooner, between 0 and 7 seconds. There were no significant effects on decision time $(F(2,135)=.312, p=. .733)$ or perceived effort $(F(2,135)=0.81, p=.445)$. Thus, hypotheses $\mathrm{H} 1, \mathrm{H} 2$ and $\mathrm{H} 3$ are supported.

\section{Insert Table 2 here}

The number of pages accessed was not significantly different in the delayed treatments $(F(2,135)=2.88, p=0.060) ; H 4$ is not supported. ANOVA analysis on the number of data points examined per page found a significant difference due to delay $(F(2,135)=3.58, p=0.031)$. A Ryan-Einot-Gabriel-Welsch $F$ test found those in the 11-second delay treatment to examine more points per page than those in other treatments, but no differences between zero-delay and 7-second delay treatments. Thus, situation normality of search behavior is present (H5 supported). Figure 2 shows the tipping point for points per page as well as number of pages. Decision quality was not related to delay $\left(X^{2}(2)=3.97, p=.138\right)(H 6$ rejected).

\section{Insert Figure 2 here}

Hypothesis $\mathrm{H7a} \& \mathrm{H} 7 \mathrm{~b}$ argues that delays will cause decision makers to reduce their search of lower importance information but will not affect the search of high importance pages. A repeated measures ANOVA found significant effects due to delay $(F(2,135)=4.62, p=.011)$, due to page importance (whether high, medium or low) $(F(2,405)=38.78, p<.001)$, 
and due to an interaction between delay and page importance $(F(4,405)=4.26, p=0.002)$. These differences were due primarily to a reduction in the number of the lowest value pages searched, although the greatest search reduction was seen for the 7-second delay treatment. This is similar to the findings by Dennis and Taylor (2006); however, our study extends their findings by examining the effects of increasing delays when examining pages with varying importance. Thus, our results show that fewer lower importance pages are searched under 7-second delays. However, the results for the 11second delay do not fit this pattern. Therefore, we conclude that $\mathrm{H} 7 \mathrm{a}$ was supported but $\mathrm{H} 7 \mathrm{~b}$ was not.

Under information foraging theory, we would expect decision makers to focus first on the high value pages, then on medium value pages, and finally on low value pages. There were four high value pages, so we examined system logs to see if the first four pages searched by each participant were high value pages (a total of 552 pages were searched by all participants). We found that, of the first four pages examined, all participants read all high value pages first except for the case in which 2 medium value pages were examined by participants in the 7-second delay treatment. There were 8 medium value pages, so we next examined the first 12 pages examined by all participants ( 4 high value +8 medium value pages), and found that over $99 \%$ of the first pages examined were high or medium value pages with only 13 low value pages (less than 1\%) searched (the low value pages were examined by 4 participants in the zero-delay treatment, 4 participants in the 7-second delay treatment (who viewed a total of 7 low value pages), and 2 participants in the 11-second delay treatment). We conclude that participants were more likely to first search high value pages, then medium value pages, and finally the low value pages, and that this pattern was not materially affected by delay.

Our step function assumption surrounding delay and search behavior (i.e. a tipping point) appears to be relevant for experiment 2 as well. As described above, there were no significant behavioral changes found between the 0 -second and 7-second delays. It was not until the 11-second delay was present in which we found significant changes in user search behaviors. This would suggest that perceptions of delay changes between a 7 and 11 second delay which triggers change in search behaviors; as this tipping point is passed, information "foragers" change the number of points examined per page. Past this tipping point (i.e. 11-second delay), foragers examined more points per page.

\section{GENERAL DISCUSSION}

Information foraging theory posits that information seekers will modify their information search strategies in the face of increasing search costs in order to maximize their rate of gaining valuable information per unit of search cost. Like its 
wildlife counterpart, the information forager will tend to engage in "within-patch" foraging until some point where the perceived benefits of moving to and subsequently, foraging in a new patch of information outweighs the cost of moving between patches. In both of our experiments, participants' search behavior remained unchanged in low delay situations because the situation was perceived to be normal. Once response time delays increased beyond the point of being normal, the cost to move between Web pages induced participants to search fewer Web pages in total (experiment 1) or fewer lower importance Web pages (experiment 2), and in both cases induced them to examine more information within each page (see Table 3 for a summary of results).

\section{Insert Table 3 here}

Participants in our experiments perceived delays fairly accurately, except the 11-second delay. In both experiments, participants perceived the delay noticeably less than the actual 11 seconds ( 7.5 seconds and 7.0 seconds). Satisfaction decreased as delay increased, but not drastically; from no delay to an 11 -second delay, satisfaction dropped by $20 \%$ in Experiment 1 and $13 \%$ in Experiment 2. Thus, we conclude that the delays in our experiments were noticeable, but tolerable. This suggests that the conventional wisdom about the length of tolerable delays being about $7-11$ seconds is appropriate at least for the tasks and participants in our studies. While we would all prefer no delays in our Web searches, we tolerate some modest delay with little impact on our satisfaction as we perceive them to be normal. Once such tolerable delays increase to the point that they are no longer seen as normal, they can induce us to change the way we search.

Our data suggest that the impact of delay is non-linear. That is, there is not some gradual and incremental change in behavior as delay slowly increases. These findings go beyond prior research in which the presence or absence of delays alone is posited to affect user behavior (Dennis \& Taylor, 2006). Our results suggest that situation normality impacts search behavior as a step function: delay has little impact until it reaches some tipping point, in which users' perceptions of delays change, triggering a change in search behavior. For the participants, Web pages, and tasks in these two experiments, behavior changed as delay increased from 7 seconds to 11 seconds, suggesting that the tipping point lies somewhere in the 8-11 second range. This tipping point, of course, only applies for the conditions used in these experiments. It is also quite likely that as delay increases farther, it might reach another tipping point which results in abandoned search (Fui Hoon Nah \& Kim, 2000). 
We conclude information foraging theory explains search behavior on the Web in the face of tolerable delays with situation normality explaining why these delays have a step function effect on search behavior. Faced with tolerable delays, the pattern of results consistently suggests, across both experiments, that users tend to act as information foragers. As delays increase beyond what is normal, users increase the depth of search within pages and reduce the breadth of search by examining fewer pages (lower importance pages, if possible) so that they do not spend more time in the search for information (as illustrated in Figure 3). While greater within page search in the face of increasing delays may seem counterintuitive, we believe this behavior is frequently encountered.

\section{Insert Figure 3 here}

Tolerable delays affected the nature of information search by increasing the depth of search and decreasing the breadth of search. The net impact of this change was not necessarily an overall decrease in search as found in prior research in non-Web environments (Benbasat \& Todd, 1996; Karim et al., 1998; Smith et al., 1978; Teeni, 1990; Vessey, 1994). In Experiment 1, participants in 11 second delay treatment examined more data points in total (167 points vs. 145, 137 , and 137 points for the 0,4 , and 7 second delay treatments, respectively), but this difference was not significant $(F(3$, $119)=0.70, p=.555)$. In Experiment 2, the 11 second delay again induced the greatest total information search (185 points vs. 148 and 137 points for the 0 and 7 second delay treatments, respectively) and the difference was statistically significant $(F(2,135)=3.70, p=.027)$.

Thus, counter to prior research in non-Web environments, our results show that in multi-page Web environments, delay did not reduce total information search (experiment 1) but actually increased it (experiment 2). Thus, the total volume of search as shown in Figure 1 may be greater or lesser after reaching the delay tipping point; it depends upon the way in which delay decreases the number of pages and increases the number of points within each page. One of the important differences between the experiments was that in Experiment 2, the relative importance of different Web pages was clear; in Experiment 1, because the pages were organized by alternative not attribute, participants could not choose to omit lower importance pages. This greater ability to shape the breadth and depth of the information search in Experiment 2 was likely one reason why the differences in total search and the number of pages differed between treatments.

These observations may also be viewed from a risk perspective. Risk is a function of the probability of occurrence and the consequence of the occurrence (i.e., Risk = Probability x Consequence) (Rainer, Snyder, \& Carr, 1991). Each 
movement from one page to the next represents a gamble that information not considered or overlooked on the page being left will not be needed. The probability of needing information on a different page is high when the number of pages (and consequently the amount of information not located on the currently viewed page) is large as was the case here. Given that the consequence (delays from backtracking and relocating information) cannot be controlled, spending more time examining information on each page is a natural response. In essence, user behavior shifts from minimizing actual costs to minimizing opportunity costs of overlooked data.

We argued that information foragers would attempt to reduce the number of less important pages examined as delays increased. In Experiment 2, participants chose to examine all high importance pages, but examined fewer lower importance pages as delays increase. As with foragers in nature, certain "dietary elements" (i.e. high importance pages) may be seen as indispensable to information foragers, thus these elements cannot be ignored.

Interestingly, mean decision quality was extremely high for all treatments in both experiments, with no statistically significant differences even though most participants did not examine all available data. The nature of the tasks allowed for successful use of incomplete decision strategies in which the low value pages were omitted from consideration; however, if participants omitted the medium value pages, then they were likely to make incorrect choices. It also suggests the tasks proved sufficiently salient to the participants to induce them to produce high quality decisions. A higher difficulty task (e.g., a higher information load or a time limit on reaching a decision) or a less salient task may have produced different results in terms of decision quality and possibly other outcome variables. A higher information load (and consequently higher perceived effort) would likely result in greater variation in information use (number of pages and points per page) and consequently greater variation in decision time and decision quality. Likewise, a less salient task would also result in greater variation in information use, as less motivated searchers resort to non-compensatory strategies in order to reduce the effort required.

Additionally, as delay increased in both experiments, the time taken to make a decision and the perceived effort expended did not increase. We interpret this pattern to suggest that tolerable delay did not have material effects on time taken or effect expended for these tasks and participants. 


\section{Limitations}

Our study suffers from the usual limitations of experimental research. First, the results obtained here are based on a limited number of student participants and consequently may have failed to capture the full breadth of Web user characteristics such as experience level, socioeconomic status, culture and so on. Business school students may be more or less prone to be "effort minimizers" and/or "information foragers" than customers shopping over the Web. Thus, future research needs to address a broader range of individual factors.

Task characteristics may have also impacted the obtained results. The task involved a relatively high value product which may have resulted lower sensitivity to the delays imposed. The experimental incentive may have also lessened sensitivity to the delays. This reduced sensitivity may have resulted in reduced treatment effects. In order to increase statistical control, we utilized uniform delays for all information pages where in practice, caching and variations in Internet (network) delays would have resulted in a less consistent pattern.

In addition, individual characteristics were not explicitly measured but may also have had a material impact. Computer anxiety, perceived time pressure and other individual differences many have affected perceptions of time. Thus, the tipping point, and consequently search strategies for a given delay may have differed across participants.

\section{Implications for Future Research and Practice}

Notwithstanding the limitations, we believe this study has several implications for researchers and practitioners. While the tolerable delays in our study had significant but rather modest impacts on satisfaction, they did induce changes in information search behavior. Rather than simply reducing search as previously argued, tolerable delays did not decrease the total amount of information examined and even increased it in the second experiment.

The changes in search behavior seen between the 7-second and 11-second delay offers some interesting insights not considered in previous research. In our experiments, a tipping point occurs somewhere between 7 to 11 seconds that resulted in changes in search behavior. We propose this tipping point in search behavior is caused by situation normality perceptions about delays on the Internet. Searchers appear to see modest delays as normal when searching online. As long as delays do not exceed this "normal" perception (about 7 seconds in our study), behavior does not change. Once delay increases to a point beyond "normal", search behavior will change in response to delays. Further research should be 
conducted to understand how delay situation normality affects other aspects of the online experience beyond search behavior (i.e. buying behavior, repeat visits, etc.).

This has a clear implication for the design of Web sites. Although minimizing delay remains important, the step function relationship between delay and changes in behavior suggests that Web developers need not be as concerned about delay as previously thought. Minor increases in delay should have little impact on user behavior. If delay is perceived as "normal" by users, no changes in behavior occur. As long as one's Web site is not noticeably slower than one's competitors, delay should have little effect on user behavior, because it will be seen as normal. This means that investing resources to reduce delay relative to competitors is not likely to change user behavior or bring many benefits.

The avoidance of delay remains a general issue during website development, but a site wanting to leverage certain search behaviors that occur after the delay tipping point (e.g., greater depth, less breadth) may consider judiciously increasing delays to induce different user behaviors. For example, there may be opportunities to capitalize on delays as means of increasing attention to select areas of a Web site. This increased within page search seen with tolerable delays could potentially be used to improve Web page "stickiness" - as the delay to move to new pages increases, within some tolerable range, users will spend more time reading information within pages, although they will likely decrease the number of pages they visit. This has applications for decision support and Web-based training by increasing the depth of search, at least for motivated users. By selectively increasing foraging costs, web sites may be able shape users' information search.

We found this tipping point for our users, tasks and situations employed in the experiments. Our tasks involved experienced users participating in tasks of low to medium complexity. Future research is needed to identify where a tipping point occurs for different tasks including less structured, more complex searches while examining a greater variety of users to see if this tipping point can be generalized to the larger public. Additionally, research has shown that users employ a number of rules when searching for information online (c.f. Browne, Pitts, \& Wetherbe, 2007) depending upon task characteristics. The tipping point may also differ by user and context. One user may be very tolerant of delays, while another may not. The same user may be less tolerant of delays when there is an urgent task. Further studies are needed to understand the delay effects on a variety of search types, tasks and users. 
Traditionally, designers have focused on minimizing delay, and as a result, many firms offer "low-bandwidth" versions of their Web site to address the delay issue especially for mobile users. However, given the changes in information search behavior that are associated with tolerable delays, this may only address part of the problem with delays. More consideration should be given to the partitioning of information among pages because both within-page and between-page search are affected by delay. For example, in many ecommerce sites, it is both possible to see all search results including pictures or display the results one subset at a time. Depending upon type of information presentation, designers should consider how a tipping point caused by situation normality can impact Web search."

An interesting finding emerging from the current study was the results associated with information presentation (i.e. attribute vs. alternative). Prior research suggests that presentation of information can have a significant effect on information searching strategies (Yuan \& Belkin, 2010a). Our findings suggest that decision quality will vary in relation to delays when information is presented differently. Thus, in situation where delays can be minimized, an attribute based presentation may result in the best decision. Conversely, when delays are a major concern and there is a potential to have increased delays, presentation of information by alternative results in a higher decision quality when compared to attribute based presentation. Therefore, not only should delay be a concern for developers but presentation of information must also be accounted for in decision quality when delay is present.

In this study, all pages within one treatment had equal delays to create the impression of a slowly responding Web site or slower connection to the Internet. In practice, delays are not uniformly distributed because of variations in page size/complexity; some pages experience more delay than others (particularly those with many graphics). Many firms have opted for very complex home pages with animation/video downloads or java initializations that result in appreciable initial delays. Much of the research on home page complexity has been focused almost exclusively on satisfaction (Geissler, Zinkhan, \& Watson, 2001; Otto, Najdawi, \& Caron, 2000). Future research should also address the potential effect that high cost home page downloads may have on subsequent information search on the same site. Additional research is also needed to examine how overall site complexity perceptions affect information search.

The apparent chain of causation - for a given delay and task, variation in delays leads to variations in the number of pages accessed and the amount of data per page examined which in turn may affect decision quality, decision time, perceived effort, and satisfaction suggest several areas for future research. Our tasks provided moderate information load: 
16 attributes for 20 cameras. With a higher information load, we believe that participants would have been more sensitive to delays and would have been more likely to change their search behavior in response to increasing delay. This is an empirical question for future research.

The use of increasingly complex Web content and the growing popularity of lower bandwidth mobile devices promises to make delays an important design concern for some time to come. Based on our results, we believe that situational normality and information foraging theory are useful theoretical lenses for understanding the effects of tolerable delays on Web search behavior. The impact of delay on information search is more complex than originally believed. Rather than increased delay simply reducing information search as prior research might suggest, increased delay has little impact until a tipping point is reached, beyond which it changes the shape of the search (less breadth and more depth) and may even increase the amount of information searched. 


\section{Footnotes:}

1 There are however notable exceptions to this effort minimizing maxim. For example, accuracy is the objective when the results of an effort are irreversible (such as a surgeon performing brain surgery). Or similarly, when the task is particularly salient (for example, choosing the name of one's first born child) (Beach, 1978).

${ }^{2}$ For example, a customer shopping online will often minimize effort expended by focusing on the available information on the product given the time he or she has available for shopping. While there may be exceptions, generally, effort will remain constant throughout the task while other things may change such as quality and search strategy.

${ }^{3}$ Although caching technologies such as browser caching or proxy server solutions can mitigate delays in successive retrievals of the same page, some pages must be downloaded anew because they have short expiration periods or cannot be cached due to search results, security settings or limited memory, especially in handheld mobile devices. Furthermore, caching only addresses delays that are a function of network download rather than the page rendering process.

${ }^{4}$ The 0 second delay manipulation represents the treatment in which no delay was imposed. This does not mean the complete absence of delay but only nominal delay will be present (e.g. 0.5 seconds). 


\section{REFERENCES}

Ahituv, N., Igbaria, M., \& Sella, A. (1998). The effects of time pressure and completeness of information on decision making. Journal of Management Information Systems, 15(2), 153172.

Baier, A. (1986). Trust and Antitrust. Ethics, 96(2), 231-260.

Bates, M. J. (1989). The design of browsing and berrypicking techniques for the online search interface. Online Information Review, 13(5), 407-424.

Bates, M. J. (2002). Toward an Integrated Model of Information Seeking and Searching, Fourth international Conference on Information Needs, Seeking and Use in Different Contexts (Vol. 3, pp. 1-15). Lisbon Portugal: New Review of Information Behaviour Research.

Beach, L., \& Mitchell, T. (1978). A contingency model for the selection of decision strategies. Academy of Management Journal, 3(3), 439-449.

Bellman, S., Lohse, G. L., \& Johnson, E. J. (1999). Predictors of online buying behavior. Communications of the ACM, 42(12), 32-38.

Benbasat, I., \& Dexter, A. (1986). An investigation of the effectiveness of color and graphical information presentation under varying time constraints. MIS Quarterly, 10(1), 59-83.

Benbasat, I., \& Todd, P. (1996). The effects of decision support and task contingencies on model formulation: A cognitive perspective. Decision Support Systems, 17(4), 241-252.

Bhatti, N., Bouch, A., \& Kuchinsky, A. (2000). Integrating user-perceived quality into Web server design. Computer Networks, 33(1-6), 1-16.

Bouch, A., Kuchinsky, A., \& Bhatti, N. (2000). Quality is in the Eye of the Beholder: Meeting Users' Requirements for Internet Quality of Service, CHI 2000 (pp. 297-304). The Hague, The Netherlands.

Browne, G., Pitts, M., \& Wetherbe, J. (2007). Cognitive Stopping Rules for Terminating Information Search in Online Tasks. MIS Quarterly, 31(1), 89-104.

Card, S., Moran, T., \& Newell, A. (1983). The psychology of human-computer interaction. Hillsdale, N.J.: L. Erlbaum Associates.

Ceaparu, I., Lazar, J., Bessiere, K., Robinson, J., \& Schneiderman, B. (2004). Determining causes and severity of end-user frustration. International Journal of Human-Computer Interaction, 17(3), 333-356.

Charland, A., \& Leroux, B. (2011). Mobile Application Development: Web vs. Native. Communications of the ACM, 54(5), 49-53. 
Cole, C. (2011). A theory of information need for information retrieval that connects information to knowledge. Journal of the American Society for Information Science and Technology, 62(7), 1216-1231.

Cook, G. J. (1993). An empirical investigation of information search strategies with implications for decision support system design. Decision Sciences, 24(3), 683-697.

Davis, E. S., \& Hantula, D. A. (2001). The effects of download delay on performance and end-user satisfaction in an Internet tutorial. Computers in Human Behavior, 17(3), 249-268.

Davis, G. B., \& Olson, M. H. (1985). Management information systems : conceptual foundations, structure, and development (2nd ed.). New York: McGraw-Hill.

Dellaert, B. G. C., \& Kahn, B. E. (1999). How tolerable is delay: Consumer's evaluations of Internet Web sites after waiting. Journal of Interactive Marketing, 13(1), 41-54.

Dennis, A. R., \& Taylor, N. J. (2006). Information foraging on the web: The effects of "acceptable" Internet delays on multi-page information search behavior. Decision Support Systems, 42, 810-824.

Dervin, B., Foreman-Wernet, L., \& Lauterbach, E. (2003). Sense-making Methodology Reader: Selected Writings of Brenda Dervin. Creskill, NJ: Hampton Press.

DiClemente, D. F., \& Hantula, D. A. (2003). Optimal Foraging Online: Increasing Sensitivity to Delay. Psychology \& Marketing, 20(9), 785-809.

Durrande-Moreau, A. (1999). Waiting for service: ten years of empirical research. International Journal of Sercive Industry Management, 10(2), 171-189.

Edland, A., \& Svensen, O. (1993). Judgment and decision making under time pressure. In O. Svensen \& J. A. Maule (Eds.), Time pressure and stress in human judgment and decision making (pp. 27-40). New York: Plenum.

Einhorn, H. J., Hogarth, R. M., Demski, J. S., \& Swieringa, R. J. (1981). Behavioral Decision Theory: Processes of Judgment and Choice/Discussion of Behavioral Decision Theory. Journal of Accounting Research, 19(1), 1.

Fisher, R. J., \& Ackerman, D. (1998). The effects of recognition and group need on volunteerism: A social norm perspective. Journal of Consumer Research, 25(3), 262-275.

Forrester. (2007). The Big, Ugly Obstacle Holding Back eCommerce. Cambridge, MA.

Forrester. (2009). An Updated Look At Consumer Reaction To A Poor Online Shopping Experience. Cambridge, MA.

Galletta, D. F., Henry, R., McCoy, S., \& Polak, P. (2004). Web site delays: How tolerant are users? Journal of the Association for Information Systems, 5(1), 1-28. 
Galletta, D. F., Henry, R. M., McCoy, S., \& Polak, P. (2006). When the Wait Isn't So Bad: The Interacting Effects of Website Delay, Familiarity, and Breadth. Information Systems Research, 17, 20-37.

Garfinkel, H. (1963). A conception of, and experiments with, "trust" as a condition of stable concerted actions. In O. Harvey (Ed.), Motivation and social interaction: cognitive determinants. New York: Ronald Press Co.

Geissler, G., Zinkhan, G., \& Watson, R. T. (2001). Web home page complexity and communication effectiveness. Journal of the Association for Information Systems, 2(2), 1-46.

Hantula, D. A., Brockman, D. D., \& Smith, C. L. (2008). Online Shopping as Foraging: The Effects of Increasing Delays on Purchasing and Patch Residence. IEEE Transactions on Professional Communication, 51(2), 147-154.

Hong, S.-J., \& Tam, K. Y. (2006). Understanding the Adoption of Multipurpose Information Appliances: The Case of Mobile Data Services. Information Systems Research, 17(2), 162179.

Hwang, M. I. (1994). Decision making under time pressure: A model for information systems research. Information \& Management, 27(4), 197-203.

Jarvenpaa, S. L., \& Dickson, G. W. (1989). Graphics and managerial decision making: Research based guidelines. Communications of the ACM, 31(6), 764-774.

Josefek Jr., R. A., \& Kauffman, R. J. (2003). Nearing the Threshold: An Economics Approach to Pressure on Information Systems Professionals to Separate from Their Employer. Journal of Management Information Systems, 20(1), 87-122.

Kamis, A., Koufaris, M., \& Stern, T. (2008). Using an attribute-based decision support system for user-customized products online: an experimental investigation. MIS Q., 32(1), 159-177.

Karim, A. S., Hershauer, J. C., \& Perkins, W. C. (1998). A simulation of partial information use in decision making: Implications for DSS design. Decision Sciences, 29(1), 53-85.

Katz, K., Larson, B., \& Larson, R. (1991). Prescription for the waiting in line blues: entertain, enlighten and engage. Sloan Management Review, 32(2), 44-53.

Kehoe, C., Pitkow, J., Sutton, K., Aggarwal, G., \& Rogers, J. D. (1998). GVU's 10th World Wide Web User Survey. Retrieved September 23, 2004, from http://www.cc.gatech.edu/gvu/user_surveys/survey-1998-10

Khosrowpour, M., \& Herman, N. (2000). Web-enabled technologies assessment and management: Critical issues. In M. Khosrowpour (Ed.), Managing Web-enabled technologies in organizations: A global perspective (pp. 1-22). Hershey, PA: Idea Group.

Kleinmuntz, D. N., \& Schkade, D. A. (1993). Information Displays and Decision-Processes. Psychological Science, 4(4), 221-227. 
Kuhlthau, C. C. (2004). Seeking Meaning: a Process Approach to Library and Information Services. Westport, CT: Libraries Unlimited.

Leighton, T. (2009). Improving Performance on the Internet. Communications of the ACM, 52(2), 4451.

Lewis, J. D., \& Weigert, A. (1985). Trust as a Social Reality. Social Forces, 63(4), 967-985.

Lightner, N. J., Bose, I., \& Salvendy, G. (1996). What is wrong with the World-Wide Web?: a diagnosis of some problems and prescription of some remedies. Ergonomics, 39(8), 995-1004.

Lyytinen, K., \& Yoo, Y. (2002). Research Commentary: The Next Wave of Nomadic Computing. Information Systems Research, 13(4), 377-388.

McKinney, V., Yoon, K., \& Zahedi, F. (2002). The measurement of Web-customer satisfaction: An expectation and disconfirmation approach. Information Systems Reseaerch, 13(3), 296-316.

McKnight, D. H., Choudhury, V., \& Kacmar, C. (2002). Developing and Validating Trust Measures for e-Commerce: An Integrative Typology. Information Systems Research, 13(3), 334-359.

Misztal, B. (2001). Normality and Trust in Goffman's Theory of Interaction Order. Sociological Theory, 19(3), 312-324.

Moody, G., \& Galletta, D. (2008). I'm Losing Patience with your Site : The Impact of Information Scent and Time Constraints on Effort, Performance, and Attitudes. Paper presented at the Twenty Ninth International Conference on Information Systems.

Morville, P. (2005). Ambient Findability. Sebastopol, CA: O'Reilly.

Nah, F. H. (2000, May 21-24, 2000). A study of Web user's waiting time. Paper presented at the Information Resources Management International Conference, Anchorage, AK.

Nah, F. H. (2004). A study on tolerable waiting time: how long are Web users willing to wait? Behaviour and Information Technology, 23(3), 153-163.

Nah, F. H., \& Kim, K. (2000). World Wide Wait. In M. Khosrowpour (Ed.), Managing Web-enabled technologies in organizations: A global perspective (pp. 146-161). Hershey, PA: Idea Group.

Newell, A. (1990). Unified theories of cognition. Cambridge, MA: Harvard University Press.

Nielsen, J. (1993). Response times: The three important limits. Retrieved September 3, 2009, from http://www.useit.com/papers/responsetime.html

Nielsen, J. (1997). Top ten mistakes in Web design. Retrieved September 3, 2009, from http://www.useit.com/alertbox/9605.html

Otto, J. R., Najdawi, M. K., \& Caron, K. M. (2000). Web-user satisfaction: An exploratory study. Journal of End User Computing, 12(4), 3-10. 
Ozmutlu, S., Spink, A., \& Ozmutlu, H. C. (2003). Trends in multimedia Web searching: 1997-2001. Information Processing \& Management, 39(4), 611-621.

Palmer, J. W. (2002). Web site usability, design, and performance metrics. Information Systems Reseaerch, 13(2), 151-167.

Payne, J. W., Bettman, J. R., \& Johnson, E. J. (1993). The adaptive decision maker. New York: Cambridge University Press.

Pirolli, P., \& Card, S. (1999). Information foraging. Psychological Review, 106(4), 643-675.

Pollay, R. W. (1970). The structure of executive decisions and decision times. Administrative Science Quarterly, 15(4), 459-472.

Rainer, R. K., Snyder, C. A., \& Carr, H. H. (1991). Risk analysis for information technology. Journal of Management Information Systems, 8(1), 129.

Rose, G. M., Evaristo, R., \& Straub, D. (2003). Culture and consumer responses to Web download time: A four-continent study of mono and polychronism. IEEE Transactions on Engineering Management, 50(1), 31-44.

Rose, G. M., Lees, J., \& Meuter, M. L. (2001). A Refined View of Download Time Impacts on EConsumer Attitudes and Patronage Intentions Toward E-Retailers. JMM: The International Journal on Media Management, 3(2), 105-111.

Rose, G. M., Meuter, M. L., \& Curran, J. M. (2005). On-line Waiting: The Role of Download Time and Other Important Predictors on Attitude toward E-retailers. Psychology \& Marketing, 22(2), 127-151.

Rose, G. M., \& Straub, D. W. (2001). The effect of download time and consumer attitude toward the e-service retailer. e-Service Journal, 1(1), 55-78.

Saad, G. (1998). Information Reacquisition in Sequential Consumer Choice. Advances in Consumer Research, 25(1), 233-239.

Sandstrom, P. E. (1994). An optimal foraging approach to information seeking and use. Library Quarterly, 64(4), 414-449.

Schkade, D. A., \& Kleinmuntz, D. N. (1994). Information Displays and Choice Processes Differential- Effects of Organization, Form, and Sequence. Organizational Behavior and Human Decision Processes, 57(3), 319-337.

Sears, A., \& Jacko, J. A. (2000). Understanding the Relation Between Network Quality of Service and the Usability of Distributed Multimedia Documents. Human-Computer Interaction, 15(1), 43-68.

Shaw, T. (1997). Process models in information systems. In A. S. Lee, J. Liebenau \& J. I. DeGross (Eds.), Information Systems and Qualitative Research: Chapman \& Hall. 
Shneiderman, B. (1983). Direct manipulation: A step beyond programming languages. Computer, 16(8), 57-69.

Sliwa, C. (1999, September 5). Web sites battle slow download times. Computerworld, 33, 8.

Slone, D. J. (2007). The impact of time constraints on Internet and Web use. Journal of the American Society for Information Science and Technology, 58(4), 508-517.

Smith, C., \& Hantula, D. (2003). Pricing effects on foraging in a simulated Internet shopping mall. Journal of Economic Psychology, 24(5), 653-674.

Smith, J. F., Mitchell, T. R., \& Beach, L. R. (1978). A cost-benefit mechanism for selecting problemsolving strategies - some extensions and empirical tests. Organizational Behavior and Human Performance, 29(3), 370-396.

Spink, A., \& Cole, C. (2006). Human information behavior: Integrating diverse approaches and information use. Journal of the American Society for Information Science and Technology, 57(1), 25-35.

Sundar, S. S., Knobloch-Westerwick, S., \& Hastall, M. R. (2007). News cues: Information scent and cognitive heuristics. Journal of the American Society for Information Science and Technology, 58(3), 366-378.

Teeni, D. (1990). Perceived Costs and Benefits As Determinants of User Behavior - an Experiment With Matchmaking. Behaviour \& Information Technology, 9(1), 31-45.

Todd, P., \& Benbasat, I. (1992). The use of information in decision-making: An experimental investigation of the impact of computer-based decision aids. MIS Quarterly, 16(3), 373-393.

Todd, P., \& Benbasat, I. (1999). Evaluating the Impact of DSS, Cognitive Effort, and Incentives on Strategy Selection. Information Systems Research, 10(4), 357-377.

Trimmel, M., Meixner-Pendleton, M., \& Haring, S. (2003). Stress Response Caused by System Response Time when Searching for Information on the Internet. Human Factors: The Journal of the Human Factors and Ergonomics Society, 45(4), 615-622.

Vessey, I. (1994). The effect of information presentation on decision making: A cost-benefit analysis. Information \& Management, 27(2), 103-117.

Weinberg, B. D. (2000). Don't keep your Internet customers waiting too long at the virtual front door. Journal of Interactive Marketing, 14(1), 30-39.

Wilson, T. D. (1981). On user studies and information needs. Journal of Documentation, 37(1), 3-15.

Wilson, T. D. (2000). Human Information Behavior. Informing Science, 3(2).

Wilson, T. D. (2006). Revisiting user studies and information needs. Journal of Documentation, 62(6), 680-684. 
Yoo, Y. (2010). Computing in Everyday Life: A Call for Research on Experiantial Computing. MIS Quarterly, 34(2), 213-231.

Yuan, X., \& Belkin, N. J. (2010a). Evaluating an integrated system supporting multiple informationseeking strategies. Journal of the American Society for Information Science and Technology, 61(10), 1987-2010.

Yuan, X., \& Belkin, N. J. (2010b). Investigating information retrieval support techniques for different information-seeking strategies. Journal of the American Society for Information Science and Technology, 61(8), 1543-1563. 
APPENDIX 1: DIGITAL CAMERA ATTRIBUTES AND ASSIGNED IMPORTANCE LEVELS

\begin{tabular}{lll}
\hline Importance Weight & Attributes & \\
\hline & Storage Capacity & \\
High Value (70\%) & Versatility & \\
& Resolution & \\
& Print Quality & Popularity \\
& Battery Type & Warranty \\
Medium Value (20\%) & Battery Life & Flash Range \\
& Optical Zoom & Price \\
& LCD Screen & \\
\hline & Audio Recording and Playback & \\
Low Value (5\%) & Video Capacity & \\
& Freeware Photo Editing Software & \\
& Weight & \\
\hline
\end{tabular}




\section{APPENDIX 2: TASK}

For this problem, you have been asked to select a digital camera for the department. Because the camera will be used to produce content for both the department's Web site and printed media, certain camera features are more important than others. The categories and their relative weighting are as described below.

\section{Critical features (70\%):}

Because it is the desire of the department to take the best possible pictures, management has identified four factors that are very important in the selection of the digital camera (the so-called "red factors").

\begin{tabular}{|c|c|c|c|}
\hline \multicolumn{2}{|l|}{ Attribute } & Description & Evaluation \\
\hline & $\begin{array}{l}\text { Storage } \\
\text { Capacity }\end{array}$ & $\begin{array}{l}\text { This is the amount of memory installed in } \\
\text { the camera. The more installed memory, } \\
\text { the more photos can be taken before having } \\
\text { to delete or upload old photos to make room } \\
\text { for new ones. }\end{array}$ & Higher is better \\
\hline 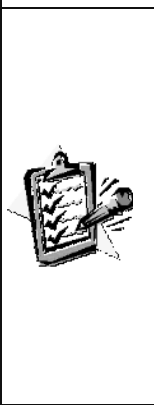 & Versatility & $\begin{array}{l}\text { Flexibility in camera settings rated by a } \\
\text { panel of experts on a scale of excellent } \\
\text { (highest), very good, good, fair, and poor } \\
\text { (lowest). This number includes a wide range } \\
\text { of adjustments for best photographs } \\
\text { including built-in exposure programs such } \\
\text { as night shooting, action, or backlight (to } \\
\text { compensate, for example, for the effects of } \\
\text { sun behind a subject). }\end{array}$ & Higher is better \\
\hline & Resolution & $\begin{array}{l}\text { The resolution (expressed in megapixels) is } \\
\text { the number of million picture elements } \\
\text { (pixels) the image sensor has. The higher } \\
\text { the number of megapixels, the greater detail } \\
\text { the camera can capture. }\end{array}$ & Higher is better \\
\hline 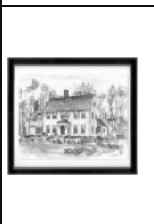 & Picture Quality & $\begin{array}{l}\text { Reflects the judgments by trained panelists } \\
\text { of glossy } 8 \times 10 \text { photos made on a high } \\
\text { quality inkjet printer. Rated as either } \\
\text { excellent (highest), very good, good, fair, or } \\
\text { poor (lowest). }\end{array}$ & Excellent is best \\
\hline
\end{tabular}




\section{Important features (25\%):}

In addition, the management has determined that eight additional factors, although not as critical as the four mentioned above, are very important in the selection process. These are:

\begin{tabular}{|c|c|c|c|}
\hline Attribute & & Description & Evaluation \\
\hline & Battery Type & $\begin{array}{l}\text { Batteries can be rechargeable or non- } \\
\text { rechargeable. Because this camera will be used } \\
\text { often, rechargeable batteries are the better } \\
\text { choice. }\end{array}$ & $\begin{array}{l}\text { Rechargeable is } \\
\text { better }\end{array}$ \\
\hline & Battery Life & $\begin{array}{l}\text { The number of high-resolution photos taken } \\
\text { on fully charged battery (or a fresh set of } \\
\text { alkaline batteries if the camera uses non- } \\
\text { rechargeable batteries). The longer the battery } \\
\text { life the lower the chance of missing a good } \\
\text { photo opportunity. }\end{array}$ & Higher is better \\
\hline & Optical Zoom & $\begin{array}{l}\text { Optical zoom is the ability of the camera to } \\
\text { increase the size of the resulting photo while } \\
\text { maintaining the same distance from the subject. } \\
\text { This size increase is expressed in terms of how } \\
\text { much larger the maximum size is from the } \\
\text { minimum size (e.g., } 2 X \text { is a factor of } 2 \text { ). }\end{array}$ & Higher is better \\
\hline & $\begin{array}{l}\text { LCD Screen } \\
\text { Size }\end{array}$ & $\begin{array}{l}\text { This is the size (length by width) of the display on } \\
\text { the back of the camera. Since the LCD screen is } \\
\text { used to preview/review, the larger the LCD } \\
\text { screen, the easier the images are to see. }\end{array}$ & Larger is better \\
\hline & Popularity & $\begin{array}{l}\text { This number is the sales rank based on sales } \\
\text { data from the } 3 \text { largest digital camera retailers. } \\
\text { The relative popularity of the camera is indicated } \\
\text { by the number of stars ( } 5 \text { stars maximum). }\end{array}$ & Higher is better \\
\hline & $\begin{array}{l}\text { Warranty/ } \\
\text { Service }\end{array}$ & $\begin{array}{l}\text { This is the length of the factory warranty for } \\
\text { the camera in months. }\end{array}$ & $\begin{array}{l}\text { Higher (longer) is } \\
\text { better }\end{array}$ \\
\hline & Flash Range & $\begin{array}{l}\text { Maximum distance from camera (in feet) in low } \\
\text { light setting that still results in a high quality } \\
\text { photo. Longer flash ranges minimize } \\
\text { the chances of taking a too dark photo. }\end{array}$ & Higher is better \\
\hline & Price & $\begin{array}{l}\text { This is the best price found for the unit including } \\
\text { applicable sales tax (and shipping if necessary). }\end{array}$ & Lower is better \\
\hline
\end{tabular}




\section{Less important features (5\%):}

Finally, there are several camera features that are add value to the camera but are not deemed essential (these have been referred to as the "green" features because you have been give the "green light" to purchase a camera without these features). These include:

\begin{tabular}{|c|c|c|c|}
\hline \multicolumn{2}{|l|}{ Attribute } & Description & Evaluation \\
\hline & $\begin{array}{l}\text { Audio Recording } \\
\text { and Playback }\end{array}$ & $\begin{array}{l}\text { If this feature is available, the camera } \\
\text { can capture sound while recording } \\
\text { video. }\end{array}$ & $\begin{array}{l}\text { Available is better } \\
\text { (than not available) }\end{array}$ \\
\hline & Video Capacity & $\begin{array}{l}\text { When the camera is used to record } \\
\text { video like a camcorder, this is the } \\
\text { maximum length of the video (in } \\
\text { seconds). }\end{array}$ & $\begin{array}{l}\text { Higher (longer) is } \\
\text { better }\end{array}$ \\
\hline & $\begin{array}{l}\text { Freeware Photo } \\
\text { Editing Software }\end{array}$ & $\begin{array}{l}\text { This software allows you to perform so } \\
\text { very basic editing (enlarging, cropping, } \\
\text { etc.) of your digital photos. This program } \\
\text { is public domain and can be downloaded } \\
\text { at not cost from the developer's Web site } \\
\text { but is included on CD with some camera } \\
\text { models as a convenience to its } \\
\text { customers. }\end{array}$ & $\begin{array}{l}\text { Available is better } \\
\text { (than not available) }\end{array}$ \\
\hline$\cdot \frac{1}{\sqrt{2}}$ & Weight & $\begin{array}{l}\text { This is the weight of the camera } \\
\text { including battery and memory card (in } \\
\text { ounces). }\end{array}$ & Lower is better \\
\hline
\end{tabular}

The department has charged you with the task of evaluating 20 potential digital cameras. Use the links on the Web page to see how the digital cameras scored. When you are ready to make a selection, press the button at the bottom of the page. 


\section{APPENDIX 3: MEASURES}

\section{Satisfaction}

How would you describe your feelings about using the Web site?

o very dissatisfied/very satisfied

○ very displeased/very pleased

- frustrated/contented

○ disappointed/delighted

\section{Perceived effort}

- I felt mentally tired and worn out after using the decision Web site.

- Using the decision Web site was a difficult and complex task.

- The overall mental workload I felt while using the decision Web site was low.

- Using the decision Web site was easy.

- Using the decision Web site required a lot of mental activity.

- I had to work very hard to use the decision Web site. 


\section{APPENDIX 4: WORKSHEET RELIABILITY}

While the use of concurrent verbal protocols (i.e., having subjects state aloud what they are doing as they perform the experimental task) have been advocated as a means of identifying the decision process strategies used by subjects, it is possible that concurrent verbal process tracing may distort subject performance (Todd \& Benbasat, 1987). In this study, we chose to use a less intrusive measure of subjects' decision processes, a worksheet on which subjects recorded information. This worksheet was considered less intrusive because all subjects in the pilot tests chose to use one voluntarily. Another technique was the use of computer logs, but this lacked the precision of the worksheets; when the user requested information on a particular attribute, the data for all attributes (alternatives) were displayed on a single page, and thus it was only possible to determine from the computer logs which pages were displayed, not which data points on each page were actually examined.

A pre-test was conducted to assess the reliability of using the subject worksheet as a measure of the number of data points. Using a similar selection task, eight senior undergraduates served as subjects for this pre-test and were randomly assigned into the delay or no-delay treatment. Two measures were used to assess the reliability of the information entered on the worksheet. First, the "browser" software used for the study produced a log that recorded all attribute data pages retrieved by the subjects and thus it was possible to determine which of the 14 attribute pages were examined by each subject. Second, concurrent verbal process tracing was used. After receiving instruction on using the system, subjects worked through a series of five practice questions to become accustomed to it, and then were given the actual experimental task. After subjects had completed half the practice problems, they were instructed to verbally state what information they were using as well as recording it on the worksheet (there is evidence to suggest verbal process tracing is less intrusive after subjects have some practice working with problems without verbal process tracing (Stone \& Schkade, 1991)). All pre-test sessions were audio taped. 
The information recorded on the worksheet matched the first measure (logs of pages accessed) for six of the eight subjects (75\%). In the other two cases, subjects accessed attribute pages for which no information was recorded on the worksheet. An examination of the audio-tapes revealed that in both cases, the subjects stated that there was no "interesting" information on

those pages (i.e., subjects accessed the information, but did not use it in their decisions).

The information on the worksheet matched the second measure (verbal process tracing) for six of the eight subjects $(75 \%)$. In one case, the subject verbally stated four more data points than were recorded on the worksheet, while in the other case; the subject verbally stated one less data point than was recorded on the worksheet. A total of 205 data points were recorded on the worksheets by all eight subjects, indicating there was an overall $98 \%$ match (204 out of 208 data points) between the worksheets and results of verbal process tracing. We concluded the use of the worksheets to record the number of data points had sufficient reliability. 